\title{
Direct UV Written Integrated Planar Waveguides Fabricated with 213nm Light
}

\author{
Paul C. Gow, Rex H.S. Bannerman, Christopher Holmes, James C. Gates, Peter G.R. Smith \\ Optoelectronics Research Centre, University of Southampton, Southampton, UK
}

Direct UV written planar waveguides in silica have been explored for over two decades [1]. The technique is capable of fabricating low-loss channel waveguides, couplers and Bragg gratings by translating an appropriate substrate through a focused UV beam. To date direct UV written waveguides have been primarily formed by using 244nm laser light, relying on the photosensitivity provided by germanium doping, and also typically boron. To induce sufficient refractive index change necessary to form waveguides the substrates also require hydrogen and deuterium loading prior to UV writing [2]. Not only does this require additional processing but over time the hydrogen present within the silica depletes, which can cause variation of the final written structures. Deep UV light at $213 \mathrm{~nm}$ has previously been used to inscribe strong fibre Bragg gratings (FBGs) in hydrogen-free Ge-doped fibres [3]. Here we present the first use of a $213 \mathrm{~nm}$ UV laser to write planar waveguide devices without the need for hydrogen loading.

Flame Hydrolysis Deposition (FHD) was used to deposit a silica core layer, doped with germanium and boron, onto a thermally oxidised silicon wafer. An overclad layer was formed on the core layer which was doped with phosphorus and boron. Individual planar chips with dimensions $10 \times 20 \mathrm{~mm}$ were diced from this wafer. A $5^{\text {th }}$ harmonic solid state laser operating at $213 \mathrm{~nm}$ wavelength was focused by a lens to a $1 / \mathrm{e}^{2}$ spot radius of 23 $\mu \mathrm{m}$ and the planar chip was translated through the beams focus to write waveguides into the core silica layer. For each waveguide the speed of translation was varied to change the fluence, and therefore the refractive index contrast of each waveguide. The planar chips were written directly without undergoing loading with hydrogen or deuterium. The mode size of the best resulting waveguide, written with a fluence of $19 \mathrm{~kJ} / \mathrm{cm}^{2}$ and measured at $780 \mathrm{~nm}$, had a radius of $21 \mu \mathrm{m}$ in the horizontal axis and $4 \mu \mathrm{m}$ in the vertical axis.
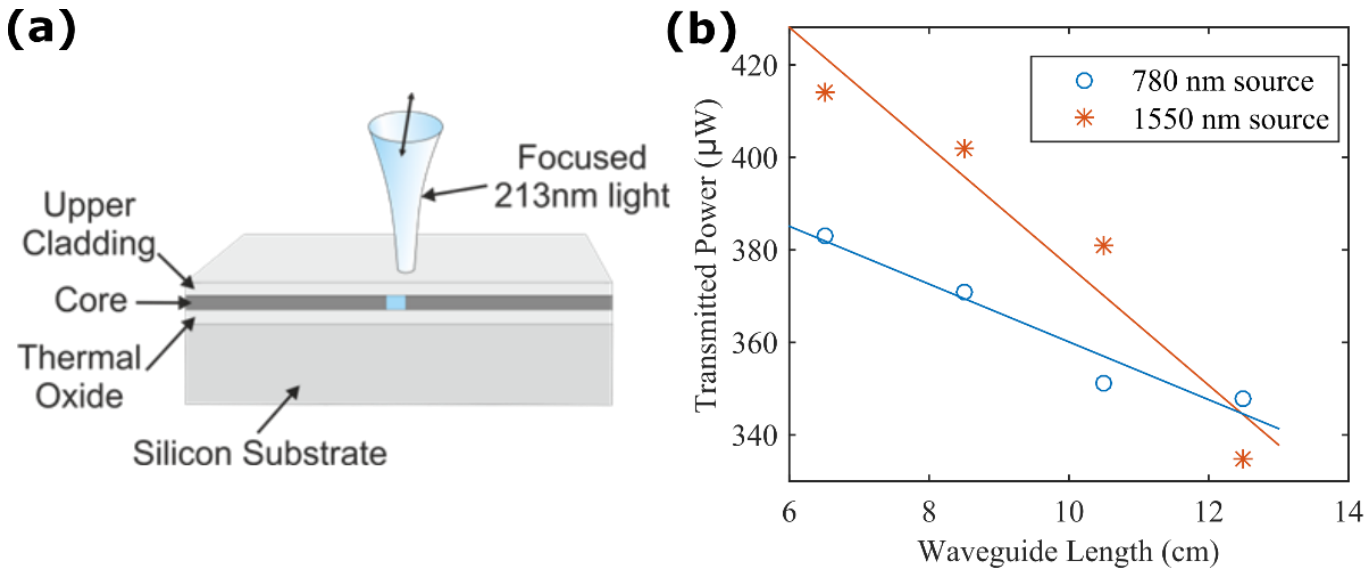

Fig. 1 (a) Schematic of the deep UV writing setup, (b) shows the transmitted power measurements with the length of waveguide for wavelengths of $780 \mathrm{~nm}$ and $1550 \mathrm{~nm}$ for the determination of propagation loss.

A planar chip measuring $10 \times 125 \mathrm{~mm}$ was diced from the same wafer and a single waveguide was written at a fluence of $15 \mathrm{~kJ} / \mathrm{cm}^{2}$. The chip was aligned with a fibre pigtail and attached using UV curing glue. The power transmitted through the waveguide was measured using a power meter for $780 \mathrm{~nm}$ and $1550 \mathrm{~nm}$ wavelengths. The waveguide was then diced to reduce its length in $2 \mathrm{~cm}$ increments, with the power measured again each time. The results shown in figure 1 (b) give a propagation loss value of $0.14 \mathrm{~dB}$ $\mathrm{cm}^{-1}$ for $1550 \mathrm{~nm}$ light and $0.06 \mathrm{~dB} \mathrm{~cm}^{-1}$ for $780 \mathrm{~nm}$ light. In the future we shall reduce the writing spot size to produce waveguides with a mode matched to standard optical fibre. We shall present our latest results of direct deep UV written waveguides, including the demonstration of single mode waveguides, detailed fluence and loss characterisation and investigation of different layer compositions.

\section{References}

[1] M. Svalgaard, "Direct writing of planar waveguide power splitters and directional couplers using a focused ultraviolet laser beam." Elec. Lett. 33, 1694 (1997).

[2] M. Fokine, and W. Marguli, "Large increase in photosensitivity through massive hydroxyl formation," Opt. Lett. 25, 302 (2000).

[3] M. Gagné, and R. Kashyap, "New nanosecond Q-switched Nd: $\mathrm{VO}_{4}$ laser fifth harmonic for fast hydrogen-free fiber Bragg gratings fabrication." Opt. Commun. 283, 5028 (2010). 